\title{
The Quality Gap in Mental Health Treatment in Australia
}

Anthony F Jorm

Centre for Mental Health, Melbourne School of Population and Global Health, University of Melbourne, Australia

\section{Corresponding author:}

Anthony F Jorm, Melbourne School of Population and Global Health, University of Melbourne, VIC 3010, Australia.

Email: ajorm@unimelb.edu.au

Key words: mental health services, antidepressants, psychological therapy, equity, evidence-based 
Australia's first National Survey of Mental Health and Wellbeing carried out in 1997 showed that mental disorders were common in Australian adults, but that many people affected did not receive treatment. Similar findings were reported during the 1990s for many other countries, leading to global concern about the 'treatment gap' in mental health care.

Since the mid-1990s, there have been considerable efforts to reduce the treatment gap in Australia. There has been a substantial increase in expenditure on mental health services, and both pharmacological and psychological treatments have had large increases in uptake (Jorm, 2014). However, despite this increase, the prevalence of mental health problems has not reduced as would have been expected. The one population mental health gain that we have had is a reduction in the male suicide rate since the late 1990s, which roughly corresponded with the introduction of the National Suicide Prevention Strategy in 1999.

It is unclear why population mental health has not improved despite increases in services and treatment. I have previously proposed that we may need to give more attention to prevention (Jorm, 2014). However, another factor may be that Australia has had an increase in the quantity but not the quality of mental health services. Here I examine recent evidence that the treatments provided in Australia are often not consistent with clinical practice guidelines and that the distribution of treatment services in the population is often less than optimal.

Using data from the second National Survey of Mental Health and Wellbeing carried out in 2007, Harris and colleagues (2015) examined the frequency and quality of treatment for people with mood or anxiety disorders. They defined evidence-based treatment as receiving pharmacotherapy (antidepressants or mood stabilizers) or psychological therapy (cognitive-behaviour therapy or psychotherapy). They also defined 'minimally adequate treatment' as either "taking an antidepressant or mood stabilizer for 1 month or longer, plus four or more consultations with any medical practitioner for mental health" or "receiving CBT or psychotherapy plus six or more consultations of 30 minutes or longer average duration with any health professionals (except a complementary or alternative medicine therapist) for mental health". Using these definitions, $39 \%$ of people with a mood or anxiety disorder sought professional help, $26 \%$ received an evidence-based treatment, and only $16 \%$ received minimally adequate treatment. In other words, less than half the people treated received minimally adequate treatment.

A similar picture emerged when data on service users' perceptions of met and unmet needs were examined. Using data from the 1997 and 2007 National Surveys, Meadows and Bobevski (2011) found that the perceived needs of service users were better met in 2007 than in 1997. However, most of the gains were in partially met need rather than fully met need, suggesting that quality of services may be lacking. 
There is also evidence that the treatments provided are not optimally targeted. While the proportion of cases receiving treatment in Australia increases with severity, according to the 2007 National Survey around 14\% of service users aged 16-64 years had no indicator of possible need, such as a current or previous mental disorder or experience of a traumatic event (Harris et al., 2014). There are also geographic inequities, with people in lower socioeconomic areas (which would have greater risk) and remote areas receiving fewer services.

There have been specific questions raised about the use of antidepressants. The use of these medications has increased rapidly despite questions about their efficacy for mild-moderate depression (Stephenson et al., 2012). The use of antidepressants for milder cases is also inconsistent with clinical guidelines that recommend psychological therapy as the first line of treatment (Harris et al., 2015). Furthermore, the age distribution of antidepressant use aligns poorly with the age distribution of mood and anxiety disorders, with antidepressants more likely to be prescribed to older people, for whom prevalence is comparatively low.

The 1990s saw increasing attention to the 'treatment gap' in mental health care. It is apparent that, while we have had some success in reducing this gap, we are now facing a 'quality gap' where a substantial proportion of people receiving services do not receive an adequate standard of treatment. Rather than continuing to persuade progressively more people (often with milder problems) into treatment, we need to focus on providing at least minimally adequate treatment to people already receiving services and to ensure that the distribution of these services is based on greatest need.

Funding

The author is supported by an NHMRC Fellowship.

Declaration of interest

None

\section{References}

Harris MG, Diminic S, Burgess PM, et al (2014) Understanding service demand for mental health among Australians aged 16 to 64 years according to their possible need for treatment. Australian and New Zealand Journal of Psychiatry 48: 838-851.

Harris MG, Hobbs MJ, Burgess PM, et al. (2015) Frequency and quality of mental health treatment for affective and anxiety disorders among Australian adults. Medical Journal of Australia 202: 185-190.

Jorm AF (2014) Why hasn't the mental health of Australians improved? The need for a national prevention strategy. Australian and New Zealand Journal of Psychiatry 48: 795-801.

Meadows GN, Bobevski I (2011) Changes in met perceived need for mental healthcare in Australia from 1997 to 2007. British Journal of Psychiatry 199: 479-484. 
Stephenson CP, Karanges E, McGregor IS (2013) Trends in the utilisation of psychotropic medications in Australia from 2000 to 2011. Australian and New Zealand Journal of Psychiatry 47: 74-87. 


\section{University Library}

\section{- M M I N E R VA A gateway to Melbourne's research publications}

Minerva Access is the Institutional Repository of The University of Melbourne

Author/s:

Jorm, AF

Title:

The quality gap in mental health treatment in Australia

Date:

2015-10-01

Citation:

Jorm, A. F. (2015). The quality gap in mental health treatment in Australia. AUSTRALIAN AND NEW ZEALAND JOURNAL OF PSYCHIATRY, 49 (10), pp.934-935. https:// doi.org/10.1177/0004867415606224.

Persistent Link:

http://hdl.handle.net/11343/91135 